\title{
Orthogonal similarity transformation of a symmetric matrix into a diagonal-plus-semiseparable one with free choice of the diagonal
}

\author{
Raf Vandebril, Ellen Van Camp, Marc Van Barel and Nicola Mastronardi
}

\begin{abstract}
Raf Vandebril
Department of Computer Science

KU Leuven, Belgium

Raf.Vandebril@cs.kuleuven.be

Ellen Van Camp

Department of Computer Science

KU Leuven, Belgium

Ellen.VanCamp@cs.kuleuven.be

Marc Van Barel

Department of Computer Science

KU Leuven, Belgium

Marc.VanBarel@cs.kuleuven.be
\end{abstract}

Nicola Mastronardi

Istituto per le Applicazioni del Calcolo

M. Picone, sez. Bari, Italy

N.Mastronardi@ba.iac.cnr.it

\begin{abstract}
In this paper we describe an orthogonal similarity transformation for transforming arbitrary symmetric matrices into a diagonal-plus-semiseparable matrix, where we can freely choose the diagonal. Very recently an algorithm was proposed for transforming arbitrary symmetric matrices into similar semiseparable ones. This reduction is strongly connected to the reduction to tridiagonal form. The class of semiseparable matrices can be considered as a subclass of the diagonalplus- semiseparable matrices. Therefore we can interpret the proposed algorithm here as an extension of the reduction to semiseparable form.
\end{abstract}

\section{Article information}

- Vandebril, Raf; Van Camp, Ellen; Van Barel, Marc; Mastronardi, Nicola. Orthogonal similarity transformation of a symmetric matrix into a diagonal-plus-semiseparable one with free choice of the diagonal, Numerische Mathematik, volume 102, issue 4, pages 709-726, 2006.

- The content of this article is identical to the content of the published paper, but without the final typesetting by the publisher.

- Journal's homepage: http://link.springer.com/journal/211

- Published version: http://dx.doi.org/10.1007/s00211-005-0665-7

- KU Leuven's repository url: https://lirias.kuleuven.be/handle/123456789/125104

\section{KU LEUVEN}


Numerische Mathematik manuscript No.

(will be inserted by the editor)

\title{
Orthogonal similarity transformation of a symmetric matrix into a diagonal-plus-semiseparable one with free choice of the diagonal ${ }^{\star}$
}

\author{
Raf Vandebril ${ }^{1}$, Ellen Van Camp ${ }^{1}$, Marc Van Barel ${ }^{1}$, Nicola \\ Mastronardi ${ }^{2}$ \\ 1 Department of Computer Science, Katholieke Universiteit Leuven, Celestijnen- \\ laan 200A B-3001 Leuven, Belgium, e-mail: Raf.Vandebril, Ellen.VanCamp, \\ Marc.VanBarel@cs.kuleuven.ac.be \\ 2 Istituto per le Applicazioni del Calcolo, CNR, via Amendola 122/D, 70126, Bari, \\ Italy, e-mail: n.mastronardi@iac.cnr.it
}

The date of receipt and acceptance will be inserted by the editor

\begin{abstract}
In this paper we describe an orthogonal similarity transformation for transforming arbitrary symmetric matrices into a diagonal-plus-semiseparable matrix, where we can freely choose the diagonal. Very recently an algorithm was proposed for transforming arbitrary symmetric matrices into similar semiseparable ones. This reduction is strongly connected to the reduction to tridiagonal form. The class of semiseparable matrices can be considered as a subclass of the diagonal-plus-semiseparable matrices. Therefore we can interpret the proposed algorithm here as an extension of the reduction to semiseparable form.
\end{abstract}

\footnotetext{
* The research was partially supported by the Research Council K.U.Leuven, project $\mathrm{OT} / 05 / 40$ (Large rank structured matrix computations), by the Fund for Scientific Research-Flanders (Belgium), projects G.0078.01 (SMA: Structured Matrices and their Applications), G.0176.02 (ANCILA: Asymptotic aNalysis of the Convergence behavior of Iterative methods in numerical Linear Algebra), G.0184.02 (CORFU: Constructive study of Orthogonal Functions) and G.0455.0 (RHPH: Riemann-Hilbert problems, random matrices and Padé-Hermite approximation), and by the Belgian Programme on Interuniversity Poles of Attraction, initiated by the Belgian State, Prime Minister's Office for Science, Technology and Culture, project IUAP V-22 (Dynamical Systems and Control: Computation, Identification \& Modelling). The scientific responsibility rests with the authors.
} 
A numerical experiment is performed comparing thereby the accuracy of this reduction algorithm with respect to the accuracy of the traditional reduction to tridiagonal form, and the reduction to semiseparable form. The experiment indicates that all three reduction algorithms are equally accurate. Moreover it is shown in the experiments that asymptotically all the three approaches have the same complexity, i.e. that they have the same factor preceding the $n^{3}$ term in the computational complexity. Finally we illustrate that special choices of the diagonal create a specific convergence behavior.

Key words Orthogonal similarity transformation - diagonal-plussemiseparable matrix - symmetric matrix

Mathematics Subject Classification (1991): Primary: 15A21, 65A15, Secondary: 15A18

\section{Introduction}

Nowadays a lot of attention is paid to the class of semiseparable and diagonal-plus-semiseparable matrices. Especially the class of diagonalplus-semiseparable matrices is very interesting as it covers also the class of semiseparable matrices, and it arises in different fields e.g. statistics $[17,11]$, the field of orthogonal rational functions [18], vibrational systems [9], time-varying systems [4]. Also different algorithms already exist for this class of matrices, ranging from solving equations with a diagonal-plus-semiseparable matrix as coefficient matrix [20], eigenvalue solvers [2,13], algorithms connected to inverse eigenvalue problems [7], inversion formulas [5,15] and also theoretical considerations connected to the eigenvalues of subblocks of these matrices exist, for example [6]. The definition and the representation of semiseparable matrices is studied in [22]. Moreover it is interesting to know that there exist reduction algorithms to transform diagonal-plus-semiseparable matrices to tridiagonal form $[8,12]$, but no algorithm exists in the other direction, i.e. there is no orthogonal reduction algorithm for transforming a symmetric tridiagonal matrix into a similar diagonal-plus-semiseparable one.

It is also well-known how any symmetric matrix can be transformed into a similar tridiagonal one $[10,16]$. This orthogonal similarity transformation forms the basic step for various algorithms. For example if one wants to compute the eigenvalues of a symmetric matrix, one can first transform it into a similar tridiagonal one and then compute the eigenvalues of this tridiagonal matrix by means of a $Q R$-algorithm $[24,25]$ or divide and conquer algorithms [3]. 
Very recently an algorithm was developed for transforming an arbitrary symmetric matrix, via orthogonal similarity transformations into a similar semiseparable one [19]. This reduction costs $9 n^{2}$ operations more than the reduction to tridiagonal form. This extra cost creates however an extra convergence behavior, namely a $Q L$-iteration step without shift is performed in each step of the reduction process on the already semiseparable part of the matrix. Hence, during the reduction, a semiseparable block whose eigenvalues are the dominant ones of the original matrix, can be split off if the gap between this cluster and the other eigenvalues is big enough. An interesting application, due to this convergence behavior, is the fact that the rank revealing factorization of a symmetric matrix can be computed using the reduction to semiseparable form ([14]). The reduction to semiseparable form, can also be used, similarly like in the tridiagonal case as a basic step for calculating for example the eigenvalues. There exist $Q R$-algorithms $[1,21]$ as well as divide-and-conquer algorithms [2, 13] to compute the eigendecomposition of symmetric semiseparable matrices.

In this paper we present an extension of the algorithm presented in [19]. The class of semiseparable matrices can be considered as a subclass of the class of diagonal-plus-semiseparable matrices, namely these matrices having as diagonal zero. The algorithm presented reduces via orthogonal similarity transformations symmetric matrices into similar diagonal-plus-semiseparable ones, with a free choice of the diagonal. Moreover, also $Q R$-algorithms exist for diagonal-plussemiseparable matrices [1] as well as divide and conquer algorithms [13]. This algorithm solves a gap in the orthogonal transformations that exists for transforming symmetric, tridiagonal, semiseparable and diagonal-plus-semiseparable matrices into each other.

The computational complexity of this reduction algorithm differs only with a term $n^{2}$ of the reduction to semiseparable form. A benefit, however, is that the reduction into diagonal-plus-semiseparable form can be seen as the application of nested $Q L$-steps with a kind of multishift depending on the chosen diagonal. Hence, the choice of the diagonal can tune the convergence behavior of the reduction algorithm. If one, for example, has already information about the spectrum, convergence behavior occurs, thereby revealing properties of the spectrum of the matrix.

The paper is organized as follows. Section 2 describes the most important part of the paper, namely the reduction to diagonal-plussemiseparable form, with a free choice of the diagonal. The computational complexity is discussed in Section 3. Section 4 describes briefly 
the convergence behavior of the reduction algorithm connected with special choices of the diagonal. Examples show that if eigenvalues appear in the top-left part of the diagonal matrix, the reduction algorithm will reveal them in the resulting diagonal-plus-semiseparable matrix. In Section 5 numerical experiments are performed comparing three reduction algorithms w.r.t. accuracy.

\section{The algorithm}

In this section, we will prove the following theorem by constructing an algorithm which fulfills the demands of the theorem.

Theorem 1 Let $A$ be an $n \times n$ arbitrary symmetric matrix and $d=$ $\left[d_{1}, d_{2}, \ldots, d_{n}\right]$ an arbitrary $n$-dimensional vector. Then there exists an orthogonal similarity transformation which transforms the symmetric matrix $A$ into a diagonal-plus-semiseparable matrix $D+S$ with $D=\operatorname{diag}(\mathrm{d})$ :

$$
Q^{T} A Q=D+S
$$

\subsection{Notation}

Before starting to construct an algorithm that transforms an arbitrary symmetric matrix into a diagonal-plus-semiseparable one, we introduce some definitions and notation.

Definition 1 When any submatrix of the upper-, or the lower-, triangular part of a symmetric matrix $S$ has rank at most one, this matrix is called a semiseparable matrix.

This kind of structure can be embedded in a lager one, more precisely:

Definition 2 A matrix has semiseparable structure of dimension $m$, when the $m \times m$ lower-right block is a semiseparable matrix. The rank of every matrix one can take out of the last $m$ columns up to and including the main diagonal must be at most one and, by symmetry, every matrix one can take out of the last $m$ rows up to and including the main diagonal has rank at most one as well. The elements belonging to this semiseparable structure are denoted by $\otimes$ : 


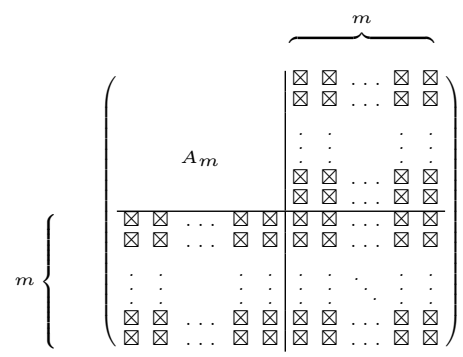

With $S_{0}^{(m)}$ we denote a matrix with semiseparable structure of dimension $m . \times$ denotes an arbitrary element of a matrix, $\otimes$ an element which we will annihilate, + a changed element and elements denoted with a $\square$ around it, belong to the semiseparable structure.

A Givens transformation working on the $i$ th and $(i+1)$ th row of a matrix, in order to annihilate an element on the $i$ th row, is denoted by $\hat{G}_{i}^{T}$ :

$$
\hat{G}_{i}^{T}\left[\begin{array}{c}
\times \\
\times
\end{array}\right]=\left[\begin{array}{l}
0 \\
\times
\end{array}\right]
$$

When $\hat{G}_{i}^{T}$ is embedded in the identity matrix, we use the notation $G_{i}^{T}$.

The algorithm proposed in this section will add at each step one row and column to the semiseparable structure of a matrix $S_{0}^{(k)}$ and one diagonal element to a matrix $D_{0}^{(k)}$. The sequence $S_{0}^{(k)}, k=$ $1, \ldots, n$ will become the semiseparable part $S$ of the requested diagonal-plus-semiseparable matrix and the sequence $D_{0}^{(k)}$ the requested diagonal part $D$.

\subsection{The first step}

Starting with the arbitrary symmetric matrix $A$, the last row and column of course form a semiseparable structure of dimension one. Even when subtracting the element $d_{1}$ of the element $A(n, n)$, the semiseparable structure remains. Hence, we can write $A$ as the sum of a matrix $S_{0}^{(1)}$ with semiseparable structure of dimension one and a diagonal matrix $D_{0}^{(1)}$ : 


$$
A=S_{0}^{(1)}+D_{0}^{(1)}:=\left(\begin{array}{ccc|c}
\times \ldots & \ldots & \bigotimes \\
\vdots & & \vdots & \vdots \\
\times \ldots & \ldots & \bigotimes \\
\hline & \ldots & \nabla & \boxplus
\end{array}\right)+\left(\begin{array}{ccc|c}
0 & \ldots & 0 & 0 \\
\vdots & \vdots & \vdots \\
0 & \ldots & 0 & 0 \\
\hline 0 & \ldots & 0 & d_{1}
\end{array}\right)
$$

with $\boxplus=A(n, n)-d_{1}$.

First all the elements of the last column of $A$ ( or of $S_{0}^{(1)}$ ) are annihilated up to row $n-2$ by means of the product of $n-2$ Givens transformations $G_{1: n-2}^{(1)^{T}}:=G_{n-2}^{(1)^{T}} \ldots G_{2}^{(1)^{T}} G_{1}^{(1)^{T}}$ and the application of $G_{1: n-2}^{(1)}$ on the columns will zero out the last row of $S_{0}^{(1)}$ up to the last but two elements:

$$
\begin{aligned}
S_{n-2}^{(1)}+D_{n-2}^{(1)} & :=G_{1: n-2}^{(1)^{T}}\left(S_{0}^{(1)}+D_{0}^{(1)}\right) G_{1: n-2}^{(1)} \\
& =\left(\begin{array}{cccc|c}
\times & \ldots & \times & \times & 0 \\
\vdots & & \vdots & \vdots & \vdots \\
\times & \ldots & \times & \times & 0 \\
\times & \ldots & \times & \times & \bigotimes \\
\hline 0 & \ldots & 0 & \otimes & \boxplus
\end{array}\right)+\left(\begin{array}{ccc|c}
0 & \ldots & 0 & 0 \\
\vdots & \vdots & \vdots \\
0 & \ldots & 0 & 0 \\
0 \ldots & \ldots & 0 \\
\hline 0 & \ldots & 0 & d_{1}
\end{array}\right) .
\end{aligned}
$$

The former transformations $G_{1: n-2}^{(1)}$ and $G_{1: n-2}^{(1)^{T}}$ did not change the matrix $D_{0}^{(1)}$.

When the next Givens transformation $G_{n-1}^{(1)^{T}}$ would be applied to $S_{n-2}^{(1)}+D_{n-2}^{(1)}$ in order to annihilate $S_{n-2}^{(1)}(n-1, n)$, the diagonal part $D_{n-2}^{(1)}$ would change. In order to avoid this, we change the matrix $D_{n-2}^{(1)}$ into the form $\hat{D}_{n-2}^{(1)}=\left[0, \ldots, 0, d_{1}, d_{1}\right]$ and hence, the matrix $S_{n-2}^{(1)}$ must be adapted into $\hat{S}_{n-2}^{(1)}$ : 


$$
\begin{aligned}
S_{n-2}^{(1)}+D_{n-2}^{(1)} & =\left(\begin{array}{cccc|c}
\times & \ldots & \times & \times & 0 \\
\vdots & & \vdots & \vdots & \vdots \\
\times & \ldots & \times & \times & 0 \\
\times & \ldots & \times & \times & \bigotimes \\
\hline 0 & \ldots & 0 & \otimes & \boxplus
\end{array}\right)+\left(\begin{array}{ccc|c}
0 & \ldots & 0 & 0 \\
\vdots & \vdots & \vdots \\
0 & \ldots & 0 & 0 \\
0 \ldots & \ldots & 0 \\
\hline 0 \ldots & \ldots & d_{1}
\end{array}\right) \\
& \left(\begin{array}{cccc|c}
\times & \ldots & \times & \times & 0 \\
\vdots & & \vdots & \vdots & \vdots \\
\times & \ldots & \times & \times & 0 \\
\times & \ldots & \times & + & \bigotimes \\
\hline 0 & \ldots & 0 & \nabla & \boxplus
\end{array}\right)+\left(\begin{array}{ccc|c}
0 & \ldots & 0 & 0 \\
\vdots & \vdots & \vdots \\
0 \ldots & \ldots & 0 & 0 \\
0 \ldots & d_{1} & 0 \\
\hline 0 \ldots & \ldots & 0 & d_{1}
\end{array}\right):=\hat{S}_{n-2}^{(1)}+\hat{D}_{n-2}^{(1)}
\end{aligned}
$$

with $+=S_{n-2}^{(1)}(n-1, n-1)-d_{1}$. So, the matrix $\hat{D}_{n-2}^{(1)}$ will not change under the similarity transformation based on $G_{n-1}^{(1)}$.

When applying now the Givens transformation $G_{n-1}^{(1)^{T}}$ on the last two rows in order to annihilate the last but one element of the last column, we get the following situation for the matrix $\hat{S}_{n-2}^{(1)}$ :

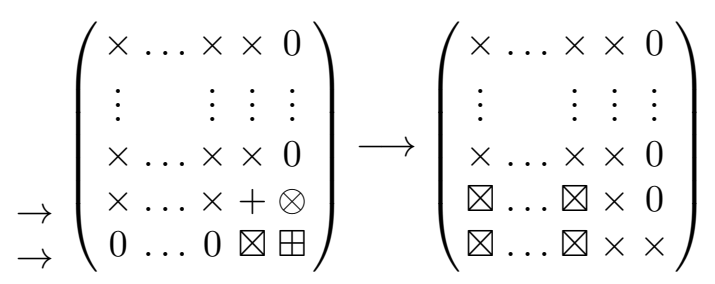

i.e., the last two rows are proportional with the exception of the entries in the last two columns.

When applying the transpose $G_{n-1}^{(1)}$ of the former transformation on the last two columns, the last two columns become linearly dependent above and on the main diagonal. Because of symmetry, the last two rows become linearly dependent below and on the main diagonal as well:

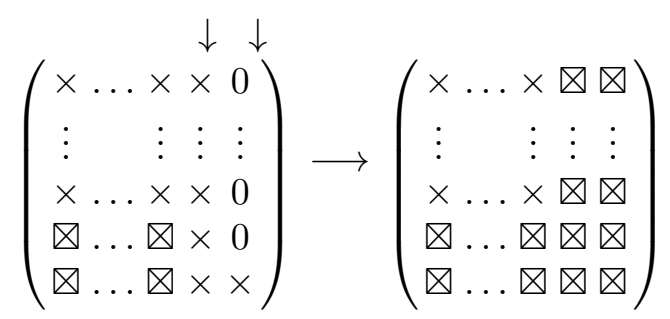

Hence, the orthogonal similarity transformation based on $G_{n-1}^{(1)}$ has transformed the matrix $\hat{S}_{n-2}^{(1)}+\hat{D}_{n-2}^{(1)}$ into a matrix $S_{n-1}^{(1)}+D_{n-1}^{(1)}:=$ 
$G_{n-1}^{(1)^{T}}\left(\hat{S}_{n-2}^{(1)}+\hat{D}_{n-2}^{(1)}\right) G_{n-1}^{(1)}$ with $S_{n-1}^{(1)}$ of semiseparable structure of dimension two and $D_{n-1}^{(1)}=\hat{D}_{n-2}^{(1)}$ the diagonal matrix $\operatorname{diag}\left[0, \ldots, 0, d_{1}, d_{1}\right]$ :

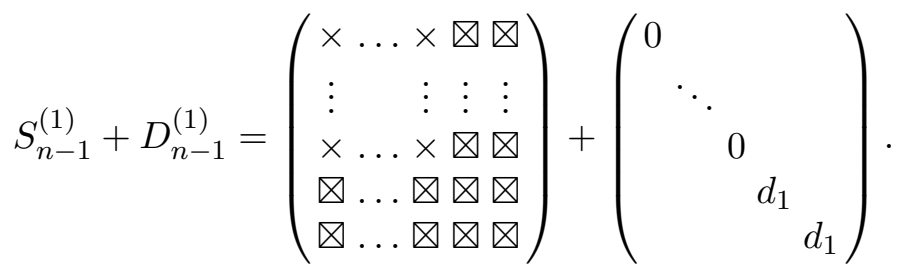

Because the element in the lower-right corner does not influence the semiseparable structure, we can change the element $S_{n-1}^{(1)}(n, n)$ into $\hat{S}_{n-1}^{(1)}(n, n)=S_{n-1}^{(1)}(n, n)+d_{1}-d_{2}$ and so $\hat{D}_{n-1}^{(1)}(n, n)$ becomes $d_{2}$. Now, the $2 \times 2$ block in the lower-right corner of $\hat{S}_{n-1}^{(1)}+\hat{D}_{n-1}^{(1)}$ will have the following structure:

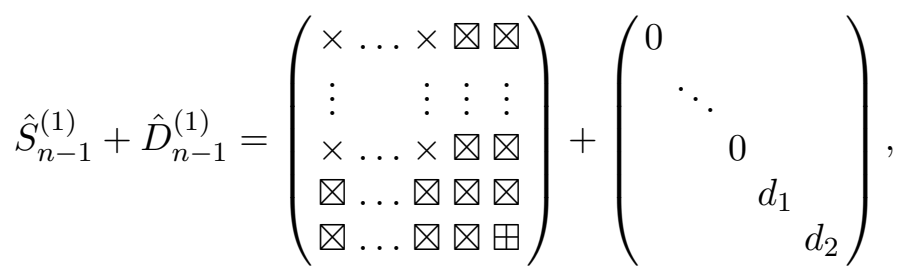

with $\boxplus$ the adapted element $\hat{S}_{n-1}^{(1)}(n, n)=S_{n-1}^{(1)}(n, n)-d_{1}+d_{2}$. At last, we put $S_{0}^{(2)}+D_{0}^{(2)}:=\hat{S}_{n-1}^{(1)}+\hat{D}_{n-1}^{(1)}$ because we have constructed the sum of a matrix with semiseparable structure 2 and of a diagonal matrix where $d_{1}$ has gone up one place and which contains the two first elements of $d$ now.

\subsection{The kth step}

Suppose that after $k-1$ steps, we have a matrix $S_{0}^{(k)}+D_{0}^{(k)}$ with $S_{0}^{(k)}$ of semiseparable structure of dimension $k$ and $D_{0}^{(k)}=\operatorname{diag}\left(\left[0, \ldots, 0, \mathrm{~d}_{1}\right.\right.$, $\left.\left.d_{2}, \ldots, d_{k-1}, d_{k}\right]\right)$ : 


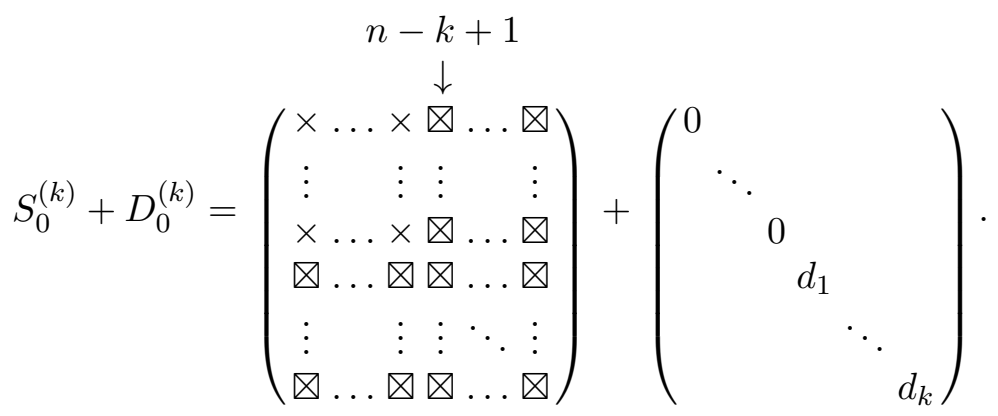

In this step we will transform $S_{0}^{(k)}+D_{0}^{(k)}$ into a matrix $S_{0}^{(k+1)}+$ $D_{0}^{(k+1)}$ with $S_{0}^{(k+1)}$ of semiseparable structure of dimension $k+1$ and $D_{0}^{(k+1)}=\operatorname{diag}\left(\left[0, \ldots, 0, \mathrm{~d}_{1}, \ldots, \mathrm{d}_{\mathrm{k}}, \mathrm{d}_{\mathrm{k}+1}\right]\right)$.

First we annihilate the elements in the $(n-k+1)$ th column up to row $n-k-1$ by means of a product $G_{1: n-k-1}^{(k)^{T}}=G_{n-k-1}^{(k)^{T}} \ldots G_{2}^{(k)^{T}} G_{1}^{(k)^{T}}$ of $n-k-1$ Givens transformations. Because of the semiseparable structure of dimension $k$, the upper-right $(n-k) \times k$ block has rank at most one. Hence, not only the elements of the $(n-k+1)$ th column will be annihilated, but also the first $n-k-1$ elements of all last $k$ columns. Applying the same transformation $G_{1: n-k-1}^{(k)}$ to the columns, the elements 1 up to $n-k-1$ of the last $k$ rows will be annihilated. Also here $G_{1: n-k-1}^{(k)}$ and its transpose do not influence $D_{0}^{(k)}$. The resulting matrix is denoted by $S_{n-k-1}^{(k)}+D_{n-k-1}^{(k)}$ :

$$
\begin{aligned}
& G_{1: n-k-1}^{(k)^{T}}\left(S_{0}^{(k)}+D_{0}^{(k)}\right) G_{1: n-k-1}^{(k)}=S_{n-k-1}^{(k)}+D_{n-k-1}^{(k)}=
\end{aligned}
$$

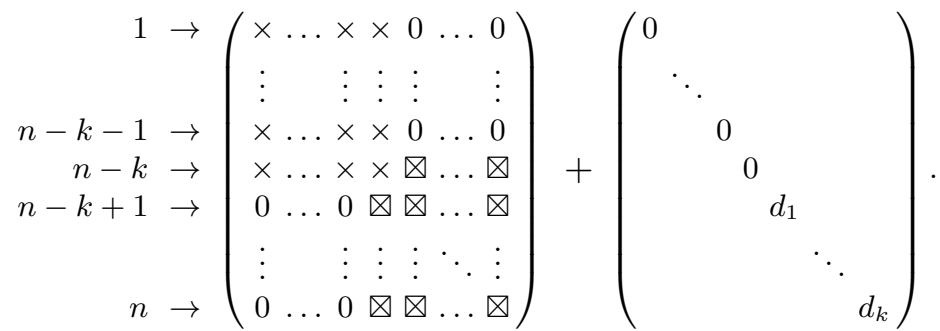

When we want to annihilate the last $k$ elements of the $(n-k)$ th row of $S_{n-k-1}^{(k)}$ by means of a Givens transformation $G_{n-k}^{(k)^{T}}, D_{n-k-1}^{(k)}$ would change. Therefore, we add $d_{1}$ to $D_{n-k-1}^{(k)}(n-k, n-k)$ and rename this as $\hat{D}_{n-k-1}^{(k)}=\operatorname{diag}\left(\left[0, \ldots, 0, \mathrm{~d}_{1}, \mathrm{~d}_{1}, \ldots, \mathrm{d}_{\mathrm{k}}\right]\right)$ such that $\hat{D}_{n-k-1}^{(k)}$ remains 
the same under the Givens transformation $G_{n-k}^{(k)}$ and its transpose. Hence, the element $S_{n-k-1}^{(k)}(n-k, n-k)$ must be adapted as follows: $S_{n-k-1}^{(k)}(n-k, n-k)-d_{1}$. This changed matrix will be denoted by $\hat{S}_{n-k-1}^{(k)}$ and we have the following situation:

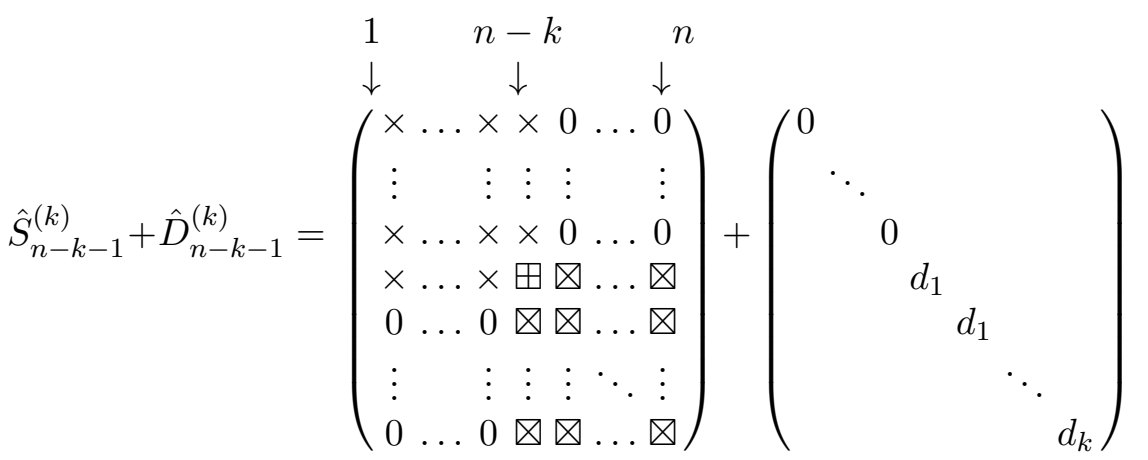

with $\boxplus=S_{n-k-1}^{(k)}(n-k, n-k)-d_{1}$. Because we changed an element on the main diagonal of $S_{n-k-1}^{(k)}$ in the upper-left corner of a semiseparable block, the dependency is not changed by this adaptation.

Now the orthogonal similarity transformation based on $G_{n-k}^{(k)}$ can be performed on $\hat{S}_{n-k-1}^{(k)}+\hat{D}_{n-k-1}^{(k)}$ :

$$
S_{n-k}^{(k)}+D_{n-k}^{(k)}:=G_{n-k}^{(k)^{T}}\left(\hat{S}_{n-k-1}^{(k)}+\hat{D}_{n-k-1}^{(k)}\right) G_{n-k}^{(k)}
$$

with $D_{n-k}^{(k)}=\hat{D}_{n-k-1}^{(k)}$. Figure 1 only shows the transformation of the matrix $\hat{S}_{n-k-1}^{(k)}$ into $S_{n-k}^{(k)}$. Remark that now also in the first elements of the $(n-k)$ th row and column appear dependencies, in contrast with the starting matrix $S_{0}^{(k)}$ where there is only dependency in the last $k$ rows and columns. This is why at the end of the $k$ th step we will have a semiseparable structure of dimension $k+1$.

Next the element on the superdiagonal at the $(n-k+1)$ th row, and because of the semiseparable structure also the other elements of that row belonging to the rank one part, should be annihilated. But once again we want the diagonal part to remain the same after the transformation, so $D_{n-k}^{(k)}$ will be changed into $\hat{D}_{n-k}^{(k)}=\operatorname{diag}\left(\left[0, \ldots, 0, \mathrm{~d}_{1}, \mathrm{~d}_{2}\right.\right.$, $\left.\left.d_{2}, \ldots, d_{k}\right]\right)$ such that the elements on the $(n-k+1)$ th and $(n-k+2)$ th 


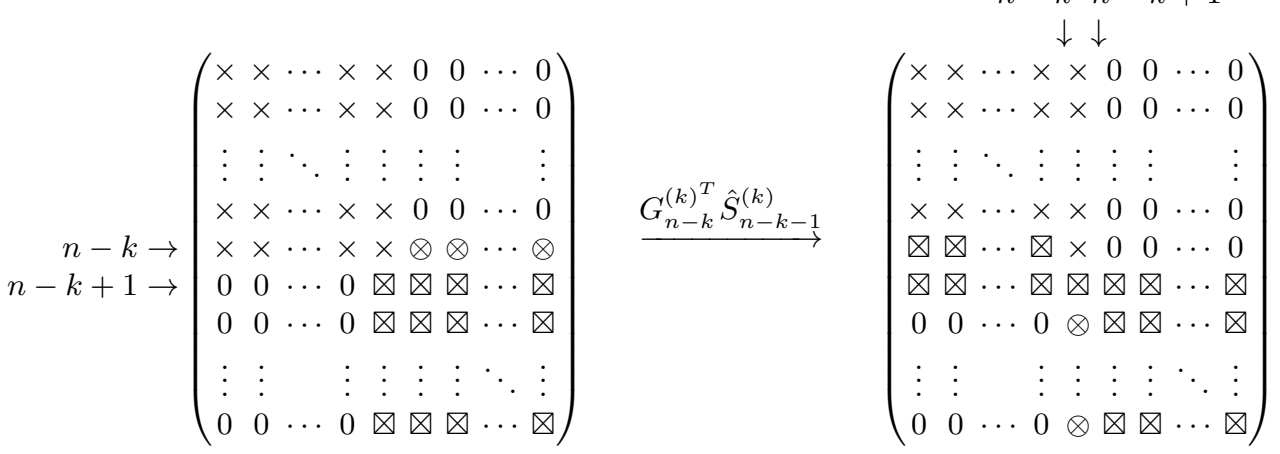

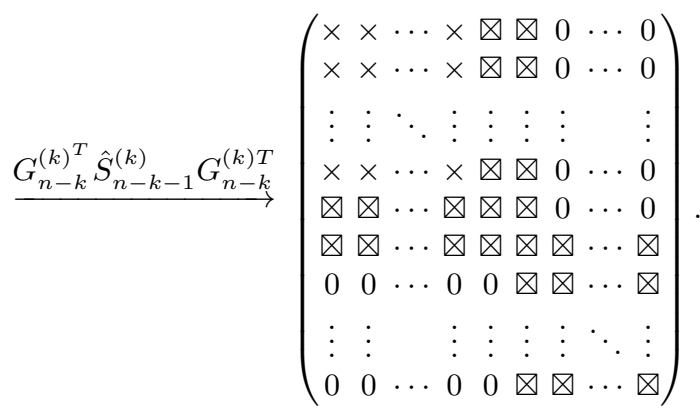

Figure 1. Transformation of $\hat{S}_{n-k-1}^{(k)}$ into $S_{n-k}^{(k)}$

row are equal. $S_{n-k}^{(k)}$ will be adapted into $\hat{S}_{n-k}^{(k)}$ too:

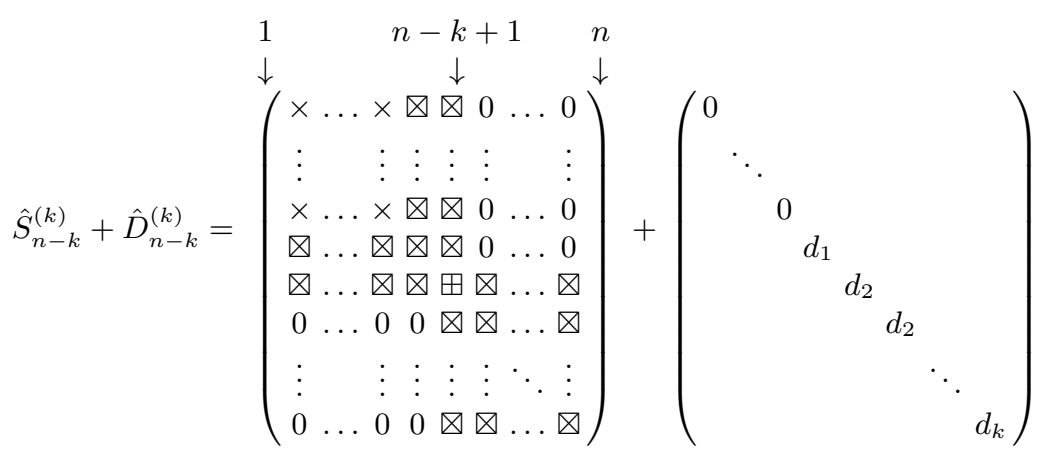

with $\boxplus=S_{n-k}^{(k)}(n-k+1, n-k+1)+d_{1}-d_{2}$. Also here this adaptation does not change the dependency because it works on the upper-left element of a semiseparable block. 
Now the Givens transformations $G_{n-k+1}^{(k)^{T}}$ and $G_{n-k+1}^{(k)}$ can be applied to $\hat{S}_{n-k}^{(k)}+\hat{D}_{n-k}^{(k)}$.

The same idea is repeated for $j=n-k+1, \ldots, n-1$. At each time, the aim is to annihilate the elements belonging to the rank one structure of the $j$ th row of $S_{j-1}^{(k)}+D_{j-1}^{(k)}$ by means of a Givens transformation $G_{j}^{(k)^{T}}$ working on the rows $j$ and $j+1$. Because we want the diagonal part $D_{j-1}^{(k)}$ to remain unchanged, the $j$ th and $(j+1)$ th element must be the same and therefore we change $D_{j-1}^{(k)}=\operatorname{diag}([0, \ldots, 0$, $\left.\left.d_{1}, \ldots, d_{l}, d_{l}, d_{l+1}, \ldots, d_{k}\right]\right)$ into $\hat{D}_{j-1}^{(k)}=\operatorname{diag}\left(\left[0, \ldots, 0, \mathrm{~d}_{1}, \ldots, \mathrm{d}_{\mathrm{l}}, \mathrm{d}_{\mathrm{l}+1}\right.\right.$, $\left.\left.d_{l+1}, \ldots, d_{k}\right]\right)$ with $l=j-n+k$. Hence, the matrix $S_{j-1}^{(k)}$ must be adapted as follows:

$$
\hat{S}_{j-1}^{(k)}(j, j)=S_{j-1}^{(k)}(j, j)+d_{l}-d_{l+1} .
$$

Because the element we adapt is the upper-left element of a semiseparable block, the existing dependencies do not change.

Next the similarity transformation based on $G_{j}^{(k)}$ can be applied in order to annihilate the last elements of the $j$ th row. But as indicated in Figure 1, the elements of the $j$ th row and column will be added to the rank one part.

$$
\begin{aligned}
& S_{j}^{(k)}+D_{j}^{(k)}:=G_{j}^{(k)^{T}}\left(\hat{S}_{j-1}^{(k)}+\hat{D}_{j-1}^{(k)}\right) G_{j}^{(k)}
\end{aligned}
$$

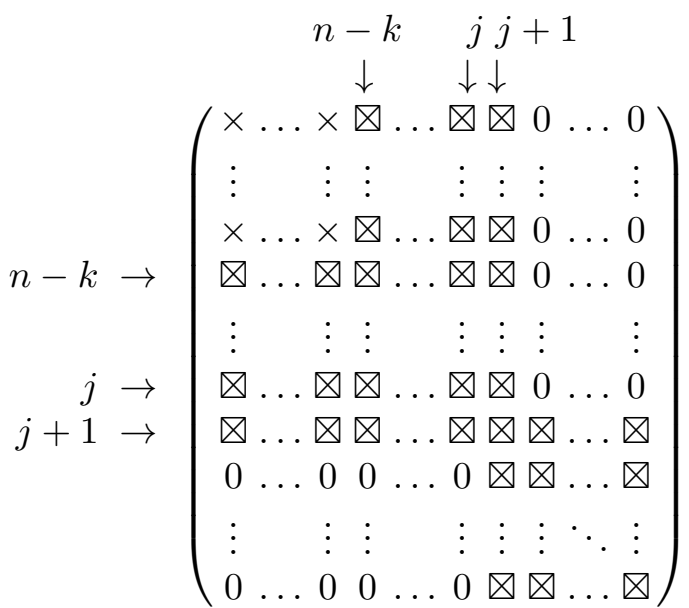

After $k-1$ similarity transformations applied in the way we just described, we will obtain that the last $k+1$ rows and columns of $S_{n-1}^{(k)}$ will belong to a semiseparable structure. 
As a last small step, the last element of $D_{n-1}^{(k)}$ will be changed into $d_{k+1}$ and $S_{n-1}^{(k)}$ will become $\hat{S}_{n-1}^{(k)}$ with $\hat{S}_{n-1}^{(k)}(n, n)=S_{n-1}^{(k)}(n, n)+d_{k}-$ $d_{k+1}$. Also here this adaptation does not influence the dependencies already existing in $S_{n-1}^{(k)}$ because only the last element on the main diagonal is changed.

Hence, we have transformed $S_{0}^{(k)}+D_{0}^{(k)}$ into a matrix $\hat{S}_{n-1}^{(k)}$ with a semiseparable structure of dimension $k+1$ and a diagonal part $\hat{D}_{n-1}^{(k)}=\operatorname{diag}\left(\left[0, \ldots, 0, \mathrm{~d}_{1}, \ldots, \mathrm{d}_{\mathrm{k}}, \mathrm{d}_{\mathrm{k}+1}\right]\right)$ as requested. So, we can define $S_{0}^{(k+1)}+D_{0}^{(k+1)}:=\hat{S}_{n-1}^{(k)}+\hat{D}_{n-1}^{(k)}$.

The next figure shows the complete 3 th step on a $6 \times 6$ matrix:

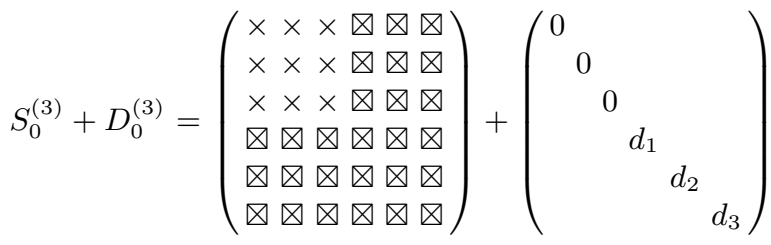

$$
\begin{aligned}
& \downarrow G_{1: 2}^{(3)}
\end{aligned}
$$

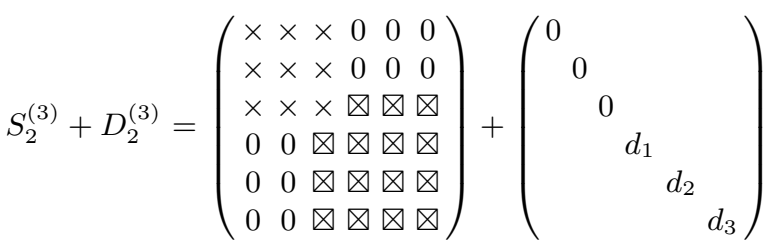

$$
\begin{aligned}
& \text { II }
\end{aligned}
$$

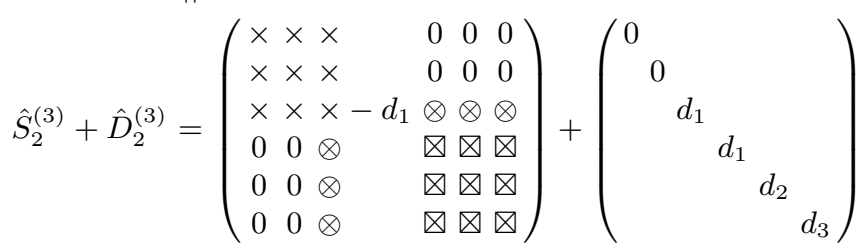

$$
\begin{aligned}
& \downarrow G_{3}^{(3)}
\end{aligned}
$$

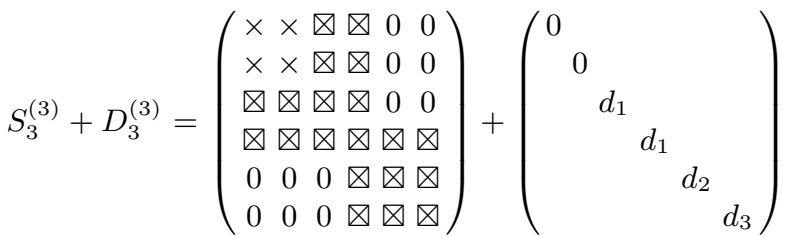




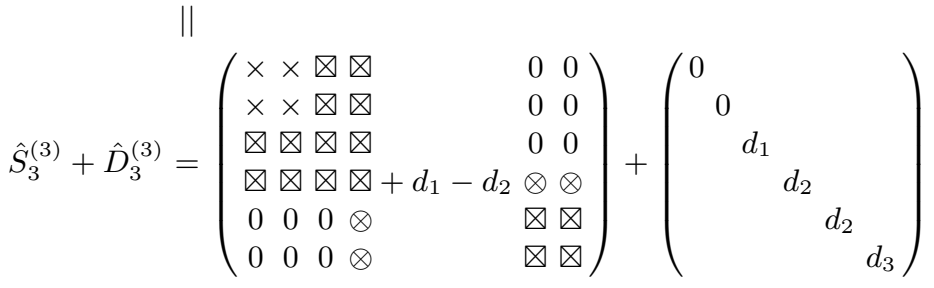

$$
\begin{aligned}
& \downarrow G_{4}^{(3)}
\end{aligned}
$$

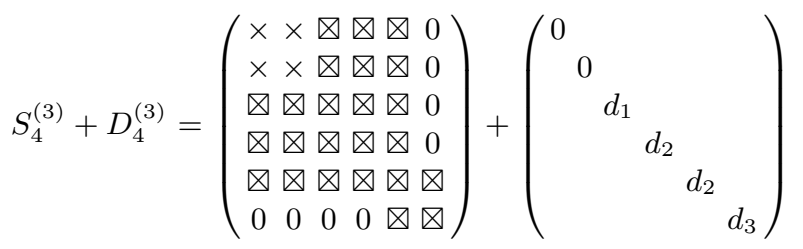

$$
\begin{aligned}
& \text { II }
\end{aligned}
$$

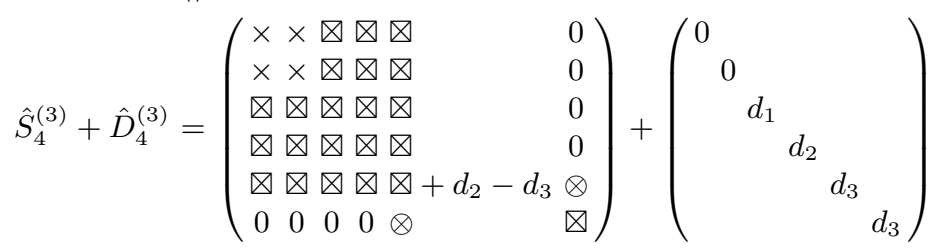

$$
\begin{aligned}
& \downarrow G_{5}^{(3)}
\end{aligned}
$$

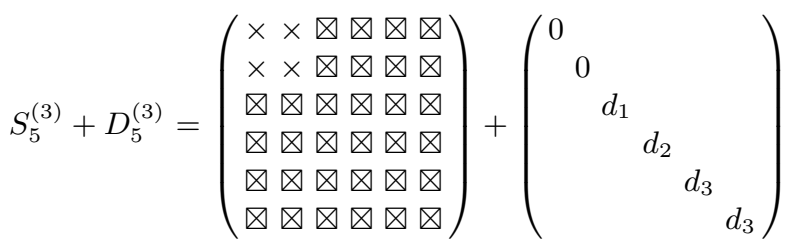

$$
\begin{aligned}
& \text { II }
\end{aligned}
$$

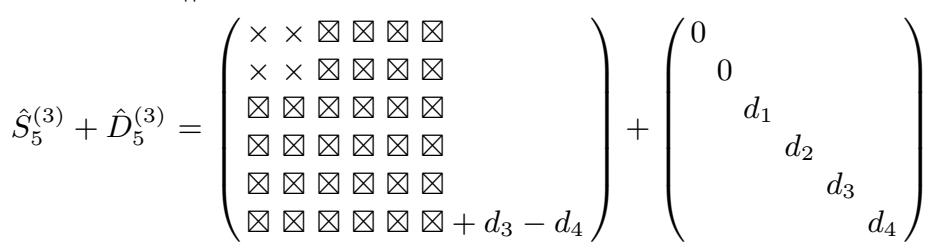

\subsection{The last step}

The last step starts with a matrix $S_{0}^{(n-1)}+D_{0}^{(n-1)}$ where $S_{0}^{(n-1)}$ has a semiseparable structure of dimension $n-1$ and $D_{0}^{(n-1)}=$ 
$\operatorname{diag}\left(\left[0, \mathrm{~d}_{1}, \ldots, \mathrm{d}_{\mathrm{n}-1}\right]\right):$

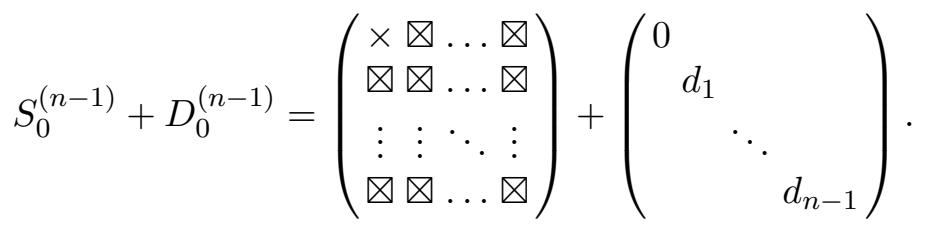

For each $j$, with $j=1, \ldots, n-1$, we want to annihilate the superdiagonal element $(j, j+1)$ of the $j$ th row, and because of the semiseparable structure of dimension $n-1$, also all elements right from the superdiagonal, by means of a Givens transformation $G_{j}^{(n-1)^{T}}$ on the $j$ th and $(j+1)$ th row. First the diagonal part $D_{j-1}^{(n-1)}$ will be updated into $\hat{D}_{j-1}^{(n-1)}=\operatorname{diag}\left(\left[\mathrm{d}_{1}, \ldots, \mathrm{d}_{\mathrm{j}}, \mathrm{d}_{\mathrm{j}}, \mathrm{d}_{\mathrm{j}+1}, \ldots, \mathrm{d}_{\mathrm{n}-1}\right]\right)$ such that $\hat{D}_{j-1}^{(n-1)}$ remains the same under the orthogonal similarity transformation based on $G_{j}^{(n-1)}$. The adaptation of $S_{j-1}^{(n-1)}$ is also as explained in the $k$ th step, more precisely, $\hat{S}_{j-1}^{(n-1)}(j, j)=S_{j-1}^{(n-1)}(j, j)+d_{j-1}-d_{j}$ if $j \neq 1$ and $\hat{S}_{j-1}^{(n-1)}(j, j)=S_{j-1}^{(n-1)}(j, j)-d_{j}$ if $j=1$. Also now the existing structure of $S_{j-1}^{(n-1)}$ is not changed. Hence, the Givens transformations $G_{j}^{(n-1)^{T}}$ and $G_{j}^{(n-1)}$ will be performed.

After these $n-1$ transformations, we have the following situation:

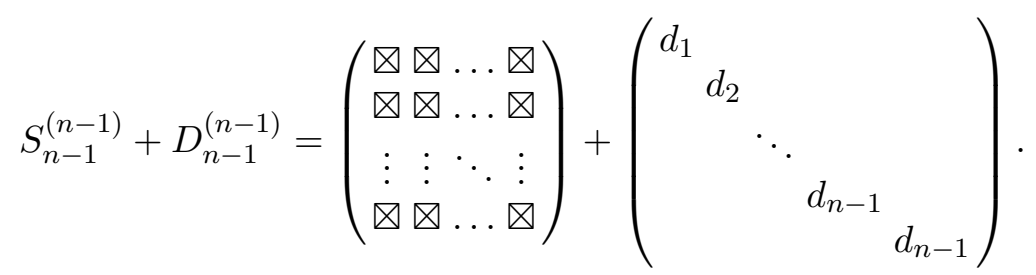

Only the last diagonal element is not of the requested form, but we can change it into $d_{n}$ and subtract it from $S_{n-1}^{(n-1)}(n, n)$. This last subtraction does not destroy the semiseparable structure and hence we have transformedwill add at each step one diagonal element to a matrix $D_{0}^{(k)}$ which will become an arbitrary symmetric matrix $A$ into a similar diagonal-plus-semiseparable one with the diagonal equal to $d$. This finishes the algorithm.

\section{Computational complexity}

The computational complexity of the orthogonal similarity reduction algorithm into diagonal-plus-semiseparable form, is of order $\mathcal{O}\left(n^{3}\right)$. 
Even more, the factor preceding $n^{3}$ is also the same as for the reduction algorithms into tridiagonal and semiseparable form.

When looking at the second highest order term, however, the computational complexity of the orthogonal similarity reduction to tridiagonal form is $9 n^{2}$ lower than to semiseparable form. This is because some extra operations need to be performed to get the semiseparable structure. For the orthogonal similarity reduction into diagonal-plus-semiseparable form not only the semiseparable structure must be restored, but also the diagonal must be adapted into the requested form. This costs $\sum_{j=1}^{n-1}(2 j+1)+1=n^{2}$ extra operations.

Figure 2 shows the computer time in seconds (the Matlab ${ }^{1}$ command cputime was used) divided by the third power of the problem size for the reduction algorithms into tridiagonal, semiseparable and diagonal-plus-semiseparable form. What one expects is that all three curves, as they have the same factor preceding the $n^{3}$ term in the computational cost, tend to the same value for large $n$. This can be observed clearly in the figure. Moreover one can see that for computational timings the extra $n^{2}$ operations performed by the reduction to diagonal-plus-semiseparable form, w.r.t. the reduction to semiseparable form are negligible.

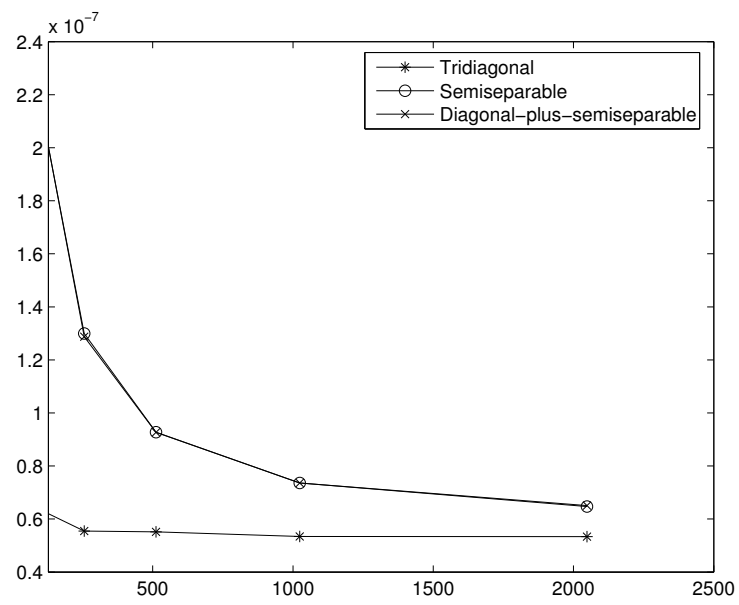

Figure 2. Computational complexity

\footnotetext{
1 Matlab is a registered trademark of the Mathwork Inc.
} 


\section{Convergence properties of the reduction algorithm.}

In this section we will briefly indicate some interesting convergence results connected with the reduction into diagonal-plus-semiseparable form.

In [19] an algorithm was proposed for reducing arbitrary symmetric matrices to semiseparable form. Moreover a detailed convergence analysis of this reduction algorithm was included. It was shown that the matrices in the reduction algorithm contained the LanczosRitz values connected to the starting vector $e_{n}$, in the part of the matrix already in semiseparable form. Moreover it was also shown that on this already reduced part at each step of the algorithm a step of a $Q L$-iteration without shift was performed. This leads to a kind of nested $Q L$-iterations performed on the matrix. An analysis of both of these convergence behaviors led to the observation that the $Q L$-iteration tended to converge to clusters if the eigenvalues of the already reduced part (these are the Lanczos-Ritz values) approximated well enough the real dominant eigenvalues of the matrix.

As the reduction to semiseparable form can be seen as a special case of the reduction to diagonal-plus-semiseparable form (we can freely choose the diagonal equal to zero), its convergence behavior is a special case of the more general convergence behavior related to the reduction to diagonal-plus-semiseparable form. The analysis of the latter reduction, however, is not so straightforward as the one in [19]. We will give here an intuitive indication of the convergence behavior. A more thorough investigation can be found in a forthcoming publication [23].

First of all, the Krylov subspace convergence behavior remains exactly the same as in the semiseparable case. This can be seen rather easily when comparing the presented reduction algorithm into diagonal-plus-semiseparable form with the reduction to tridiagonal form. The first $n-k-1$ Givens transformations performed in step $k$, in order to annihilate $n-k-1$ elements in row $n-k+1$, are exactly the same Givens transformations (up to signs), as used for creating the tridiagonal form. The remaining Givens transformations, which chase in fact the diagonal-plus-semiseparable structure downwards, do not change the eigenvalues of the lower-right $(k+1) \times(k+1)$ block. Hence, the eigenvalues of this lower-right block are the same eigenvalues as the ones appearing in the tridiagonal reduction, namely the Lanczos-Ritz values w.r.t the starting vector $e_{n}$.

Secondly, the more difficult step, is the adaptation of the proof of the nested $Q L$-iteration. We will give the idea behind this convergence property. At each step of the reduction algorithm, transforming a 
symmetric matrix into a similar diagonal-plus-semiseparable one, a kind of a $Q L$-iteration with shift is performed on the already reduced part. When having a closer look at the series of matrices $D_{0}^{(m)}+S_{0}^{(m)}$, $m=1, \ldots, n$, appearing in each step of the reduction algorithm, we notice that

$$
\begin{aligned}
D_{0}^{(1)} & =\operatorname{diag}\left(\left[0, \ldots, 0, \mathrm{~d}_{1}\right]\right) \\
D_{0}^{(2)} & =\operatorname{diag}\left(\left[0, \ldots, 0, \mathrm{~d}_{1}, \mathrm{~d}_{2}\right]\right) \\
& \cdots \\
D_{0}^{(m)} & =\operatorname{diag}\left(\left[0, \ldots, 0, \mathrm{~d}_{1}, \ldots, \mathrm{d}_{\mathrm{m}}\right]\right) \\
& \cdots \\
D_{0}^{(n)} & =\operatorname{diag}\left(\left[\mathrm{d}_{1}, \ldots, \mathrm{d}_{\mathrm{n}}\right]\right) .
\end{aligned}
$$

Hence, when applying all steps of the reduction algorithm, this can be seen as the application of a kind of $Q L$-step with a special type of shifting: on the whole matrix the shift $d_{1}$ is used, the shift $d_{2}$ is used on the whole matrix except on the first row and column, the shift $d_{3}$ on the whole matrix, except on the first and second row and column and so on....

A proof of the previous property and a detailed analysis of the chasing technique in the lower-right block is not straightforward anymore. In the reduction to semiseparable form, it could be interpreted as a $Q L$-step without shift, in the reduction to diagonal-plus-semiseparable form it should be seen as a $Q L$-step with shift.

As the analysis of these convergence behaviors and their interaction is extended, technical and detailed, we do not cover them in this publication, but in a forthcoming paper [23]. Nevertheless to illustrate the power of the convergence behavior, we show a simple consequence by some numerical experiments in Section 5. If eigenvalues of the original matrix are present in the upper-left part of the diagonal, used for the reduction to diagonal-plus-semiseparable form, then the reduction algorithm will reveal these eigenvalues.

\section{Numerical Experiments}

In this section numerical experiments are performed, using the first reduction algorithm into diagonal-plus-semiseparable form. In a first experiment the accuracy of the reduction algorithm is compared w.r.t. the orthogonal similarity reduction to tridiagonal and semiseparable form. In a second experiment the reduction algorithm is examined for special choices of the diagonal in correspondence with the second 
convergence property of Section 4, more precisely, the $Q L$-steps with shift. All the experiments are performed in Matlab and the software can be freely downloaded from the website:

http://www.cs.kuleuven.ac.be/ marc/software

For the first experiment a set of test matrices was generated of dimensions $2^{i}$ for $i=3, \ldots, 11$. The symmetric matrices were constructed in such a way that they have as eigenvalues $\left[1: 2^{i}\right]$ for each choice of $i$. In Figure 3 the relative accuracy of the eigenvalues of the reduced matrices (computed by the Matlab command eig) w.r.t. the original matrices is given. Denote the original eigenvalues with $\lambda_{i}$ and the computed ones with $\tilde{\lambda}_{i}$, then the relative accuracy is calculated as

$$
\frac{\max _{i}\left|\lambda_{i}-\tilde{\lambda}_{i}\right|}{\max _{i}\left|\lambda_{i}\right|}
$$

It can be seen clearly that all three approaches are equally accurate.

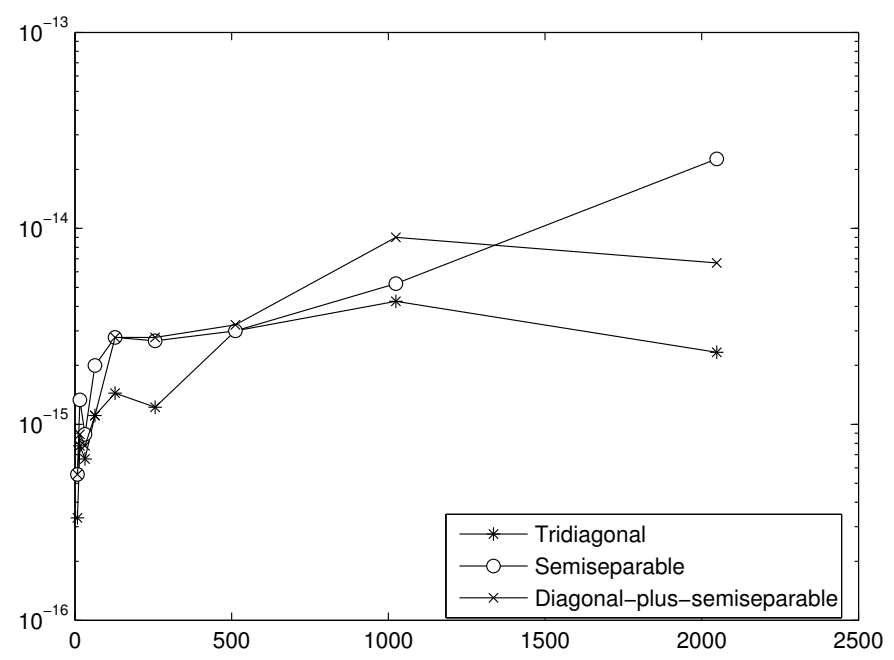

Figure 3. Relative residual of the eigenvalues.

In the second experiment we will show that if an eigenvalue is present on the top-left of the chosen diagonal, it will be revealed by the reduction algorithm. This behavior can be explained using the convergence theory from Section 4 . On the complete original matrix $A$ a step of the $Q L$-iteration with shift $d_{1}$ is performed. If $d_{1}$ is an 
eigenvalue of the original matrix the $Q L$-iteration reveals this, and we get a separated eigenvalue in the top-left position of the matrix. Secondly, on the lower-right $(n-1) \times(n-1)$ block a step of the $Q L$-iteration with shift $d_{2}$ is performed. As this submatrix contains now the remaining eigenvalues of the matrix $A$, this $Q L$-step will reveal $d_{2}$ if $d_{2}$ is again an eigenvalue. As long as the top-left elements of the diagonal, used for reducing the matrix to the appropriate form, are the eigenvalues of the original matrix, this analysis holds and all the eigenvalues will be revealed. However, if there is a diagonal element $d_{k}$ not equal to an eigenvalue, the $Q L$-iteration will not reveal an eigenvalue. Moreover if we continue our convergence analysis on the smaller lower-right $(n-k) \times(n-k)$ submatrix, the eigenvalues of this matrix are not necessary eigenvalues of the original matrix anymore. Hence, when the diagonal element $d_{k+1}$ is an eigenvalue, it will not necessarily be revealed.

To show this behavior by numerical experiments, we have created a symmetric matrix with eigenvalues $1,2,3,4,5$ and performed different reductions of this matrix to a diagonal-plus-semiseparable matrix, thereby varying the diagonal. On top the diagonal $d$ used for the reduction is shown, and below the resulting diagonal-plussemiseparable matrix. It can be seen clearly, that if an eigenvalue is present on the top-left of the chosen diagonal, it is revealed by the reduction algorithm. The reader can generate much more examples indicating this convergence behavior, as the software can be downloaded.

The last matrix shows that this behavior is related to the reduction algorithm as also a diagonal-plus-semiseparable matrix will be given, having as a diagonal eigenvalues in the upper-left positions, but the eigenvalues are not revealed.

$$
\begin{aligned}
& d=[5,4,8.3812 e-01,1.9640 e-02,6.8128 e-01] \\
& D+S=\left(\begin{array}{rrrrr}
5 & 1.0864 e-15 & -2.7746 e-15 & -1.7157 e-15 & -5.3996 e-16 \\
1.0864 e-15 & 4 & 8.8505 e-17 & 5.4728 e-17 & 1.7224 e-17 \\
-2.7746 e-15 & 8.8505 e-17 & 1.7780 e+00 & 5.8117 e-01 & 1.8291 e-01 \\
-1.7157 e-15 & 5.4728 e-17 & 5.8117 e-01 & 1.4985 e+00 & 4.6543 e-01 \\
-5.3996 e-16 & 1.7224 e-17 & 1.8291 e-01 & 4.6543 e-01 & 2.7235 e+00
\end{array}\right) \\
& d=[1,5.0281 e-01,2,3,7.0947 e-01] \\
& D+S=\left(\begin{array}{rrrrr}
1 & 3.0611 e-15 & 8.4210 e-16-3.4843 e-16 & 1.2741 e-16 \\
3.0611 e-15 & 2.2405 e+00 & 4.7803 e-01-1.9779 e-01 & 7.2327 e-02 \\
8.4210 e-16 & 4.7803 e-01 & 2.9501 e+00-3.9313 e-01 & 1.4376 e-01 \\
-3.4843 e-16 & -1.9779 e-01 & -3.9313 e-01 & 4.0160 e+00 & -3.7151 e-01 \\
1.2741 e-16 & 7.2327 e-02 & 1.4376 e-01-3.7151 e-01 & 4.7934 e+00
\end{array}\right)
\end{aligned}
$$




$$
\begin{aligned}
& d=[1,2,3,4,5] \\
& D+S=\left(\begin{array}{rrrrr}
1 & 2.3560 e-15 & 1.8142 e-15 & -9.8031 e-16 & 6.4328 e-16 \\
2.3560 e-15 & 2 & -5.9863 e-16 & 3.2347 e-16 & -2.1226 e-16 \\
1.8142 e-15 & -5.9863 e-16 & 3 & -1.1084 e-15 & 7.2736 e-16 \\
-9.8031 e-16 & 3.2347 e-16 & -1.1084 e-15 & 4 & 2.4364 e-16 \\
6.4328 e-16 & -2.1226 e-16 & 7.2736 e-16 & 2.4364 e-16 & 5
\end{array}\right)
\end{aligned}
$$

This last example shows a diagonal-plus-semiseparable matrix, not coming from the reduction algorithm. Moreover it is shown that even though the diagonal contains eigenvalues of the complete diagonalplus-semiseparable matrix in the upper-left positions, they are not revealed.

$$
\begin{gathered}
d=[1,2,3,8,7] \\
D+S=\left(\begin{array}{rrrrr}
1.3156 & -0.9067 & -0.1277 & 0.3125 & -0.0830 \\
-0.9067 & 3.7095 & 0.2408 & -0.5891 & 0.1564 \\
-0.1277 & 0.2408 & 2.7919 & 0.5091 & -0.1352 \\
0.3125 & -0.5891 & 0.5091 & 4.0448 & 1.0501 \\
-0.0830 & 0.1564 & -0.1352 & 1.0501 & 3.1381
\end{array}\right)
\end{gathered}
$$

\section{Conclusion and future work}

In this paper we presented an algorithm to transform any symmetric matrix by means of an orthogonal similarity transformation into a similar diagonal-plus-semiseparable matrix with free choice of the diagonal. When the first elements of the diagonal are eigenvalues of the original matrix, the upper-left block of the diagonal-plus-semiseparable matrix will be a diagonal block with these eigenvalues on the diagonal. This leads to some interesting convergence behaviors which will be studied in detail in a forthcoming paper.

The computational complexity is comparable with the one of the algorithms reducing into tridiagonal and semiseparable form.

\section{References}

1. D. A. Bini, L. Gemignani, and V. Y. Pan. $Q R$-like algorithms for generalized semiseparable matrices. Technical Report 1470, Department of Mathematics, University of Pisa, 2004.

2. S. Chandrasekaran and M. Gu. A divide and conquer algorithm for the eigendecomposition of symmetric block-diagonal plus semi-separable matrices. $\mathrm{Nu}$ merische Mathematik, 96(4):723-731, February 2004.

3. J. J. M. Cuppen. A divide and conquer method for the symmetric tridiagonal eigenproblem. Numerische Mathematik, 36:177-195, 1981.

4. P. Dewilde and A.-J. van der Veen. Time-varying systems and computations. Kluwer academic publishers, Boston, June 1998. 
5. Y. Eidelman and I. C. Gohberg. Inversion formulas and linear complexity algorithm for diagonal plus semiseparable matrices. Computers $\& 5$ Mathematics with Applications, 33(4):69-79, August 1996.

6. D. Fasino. Rational Krylov matrices and QR-steps on Hermitian diagonalplus-semiseparable matrices. To appear in Numerical Linear Algebra with Applications (DOI 10.1002/nla.448), 2004.

7. D. Fasino and L. Gemignani. Direct and inverse eigenvalue problems, for diagonal-plus-semiseparable matrices. Numerical Algorithms, 34:313-324, 2003.

8. D. Fasino, N. Mastronardi, and M. Van Barel. Fast and stable algorithms for reducing diagonal plus semiseparable matrices to tridiagonal and bidiagonal form. Contemporary Mathematics, 323:105-118, 2003.

9. F. R. Gantmacher and M. G. Kreĭn. Oscillation matrices and kernels and small vibrations of mechanical systems. AMS Chelsea Publishing, Providence, Rhode Island, revised edition, 2002.

10. G. H. Golub and C. F. Van Loan. Matrix Computations. The Johns Hopkins University Press, third edition, 1996.

11. F. A. Graybill. Matrices with applications in statistics. Wadsworth international group, Belmont, California, 1983.

12. N. Mastronardi, S. Chandrasekaran, and S. Van Huffel. Fast and stable algorithms for reducing diagonal plus semiseparable matrices to tridiagonal and bidiagonal form. BIT, 41(1):149-157, 2003.

13. N. Mastronardi, M. Van Barel, and E. Van Camp. Divide and conquer algorithms for computing the eigendecomposition of symmetric diagonal-plussemiseparable matrices. Numerical Algorithms, 39(4):379-398, 2005.

14. N. Mastronardi, M. Van Barel, and R. Vandebril. Computing the rank revealing factorization by the semiseparable reduction. Technical Report TW418, Katholieke Universiteit Leuven, Dept. Computer Science, Celestijnenlaan 200A, 3001 Heverlee (Leuven), Belgium, May 2005.

15. G. Meurant. A review of the inverse of symmetric tridiagonal and block tridiagonal matrices. SIAM Journal on Matrix Analysis and its Applications, 13:707-728, 1992.

16. B. N. Parlett. The symmetric eigenvalue problem, volume 20 of Classics in Applied Mathematics. SIAM, Philadelphia, 1998.

17. S. N. Roy, B. G. Greenberg, and A. E. Sarhan. Evaluation of determinants, characteristic equations and their roots for a class of patterned matrices. Journal of the Royal Statistical Society. Series B. Methodological, 22:348-359, 1960.

18. M. Van Barel, D. Fasino, L. Gemignani, and N. Mastronardi. Orthogonal rational functions and diagonal plus semiseparable matrices. In F. T. Luk, editor, Advanced Signal Processing Algorithms, Architectures, and Implementations XII, volume 4791 of Proceedings of SPIE, pages 167-170, 2002.

19. M. Van Barel, R. Vandebril, and N. Mastronardi. An orthogonal similarity reduction of a matrix into semiseparable form. Report TW 360, Department of Computer Science, K.U.Leuven, Leuven, Belgium, May 2003. To appear in SIAM Journal on Matrix Analysis and its Applications.

20. E. Van Camp, N. Mastronardi, and M. Van Barel. Two fast algorithms for solving diagonal-plus-semiseparable linear systems. Journal of Computational and Applied Mathematics, 164-165:731-747, 2004.

21. R. Vandebril, M. Van Barel, and N. Mastronardi. An implicit QR algorithm for semiseparable matrices to compute the eigendecomposition of symmetric 
matrices. Report TW 367, Department of Computer Science, K.U.Leuven, Leuven, Belgium, August 2003. To appear in Numerical Linear Algebra with Applications (DOI 10.1002/nla.425).

22. R. Vandebril, M. Van Barel, and N. Mastronardi. A note on the representation and definition of semiseparable matrices. Report TW 393, Department of Computer Science, K.U.Leuven, Leuven, Belgium, May 2004. To appear in Numerical Linear Algebra with Applications (DOI 10.1002/nla.455).

23. R. Vandebril, E. Van Camp, M. Van Barel, and N. Mastronardi. On the convergence properties of the orthogonal similarity transformations to tridiagonal and semiseparable (semiseparable plus diagonal form), 2005. In preparation.

24. D. S. Watkins. On $Q R$ algorithms for the eigenvalue problem. Nato adv. sci. inst. ser. $f$ comput systems sci., 70:687-693, 1991.

25. J. H. Wilkinson. The algebraic eigenvalue problem. Numerical mathematics and scientific computation. Oxford University Press, Great Claredon Street, Oxford OX2 6DP, 1999. 